\title{
Feasibility of Close-Range Photogrammetric Models for Geographic Information System
}

\author{
Luke Zhou \\ Office of Science, Science Undergraduate Laboratory Internship Program
}

Rice University

SLAC National Accelerator Laboratory

Menlo Park, California

August 13, 2010

Prepared in partial fulfillment of the requirement of the Office of Science, Department of Energy's Science Undergraduate Laboratory Internship under the direction of Brian C. Fuss and Catherine M. LeCocq in the Metrology Department's Alignment Engineering Group at SLAC National Accelerator Laboratory.

Participant:

Signature

Research Advisors:

Signature 


\section{TABLE OF CONTENTS}

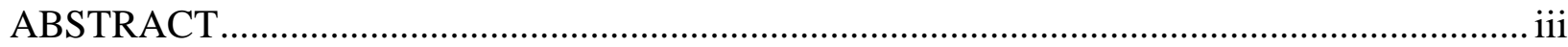

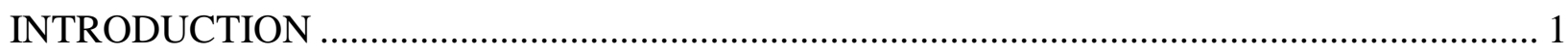

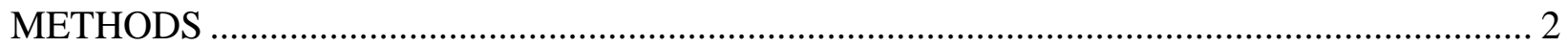

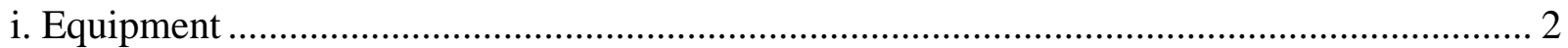

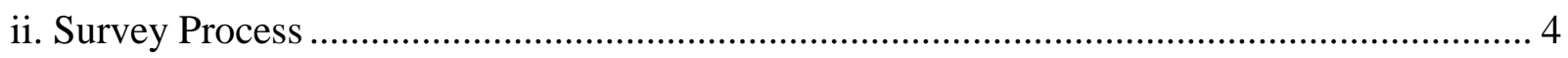

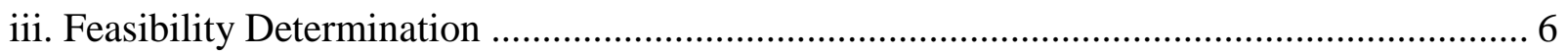

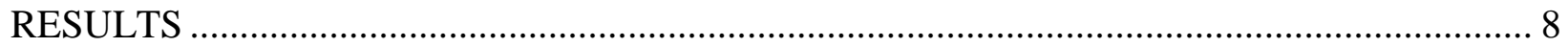

i. Equipment and Software Testing .................................................................................. 8

ii. Photogrammetry as a Replacement for Traditional Methods ................................................. 9

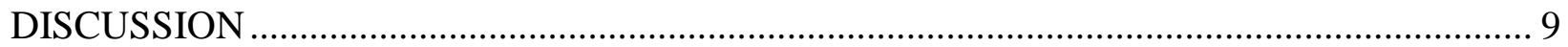

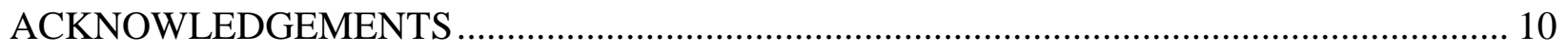

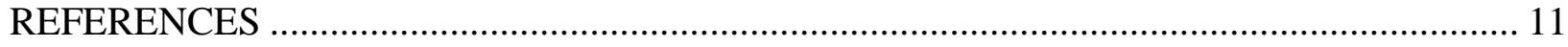

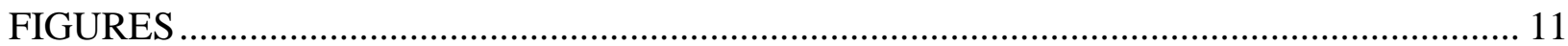




\section{ABSTRACT}

Using Close-Range Photogrammetry to Create 3D Models of As-Built Structures. LUKE ZHOU (Rice University, Houston, TX 77005), BRIAN C. FUSS and CATHERINE M. LECOCQ (SLAC National Accelerator Laboratory, Menlo Park, CA 94025).

The objective of this project was to determine the feasibility of using close-range architectural photogrammetry as an alternative three dimensional modeling technique in order to place the digital models in a geographic information system (GIS) at SLAC. With the available equipment and Australis photogrammetry software, the creation of full and accurate models of an example building, Building 281 on SLAC campus, was attempted. After conducting several equipment tests to determine the precision achievable, a complete photogrammetric survey was attempted. The dimensions of the resulting models were then compared against the true dimensions of the building. A complete building model was not evidenced to be obtainable using the current equipment and software. This failure was likely attributable to the limits of the software rather than the precision of the physical equipment. However, partial models of the building were shown to be accurate and determined to still be usable in a GIS. With further development of the photogrammetric software and survey procedure, the desired generation of a complete three dimensional model is likely still feasible. 


\section{INTRODUCTION}

Photogrammetry is a method by which images of an object are analyzed to allow for an indirect approach to measuring the size, shape, and position of the object [1]. Combining photogrammetric principles with a computer graphics process makes the construction of accurate three dimensional models of the object possible [1]. This can be done by extracting the 3D coordinates from a set of photographic images taken from all around the object using a photogrammetric software package.

The focus of this project was to test the feasibility of using photogrammetry in the Metrology Department at SLAC National Accelerator Laboratory for the purpose of measuring the dimensions of buildings on the campus to add three dimensional models of the buildings to the SLAC geographic information system (GIS). The GIS will be used to answer geographic queries about various building, utilities and experimental apparatuses within the building. To create a correct base map of SLAC campus, accurate models of the buildings are necessary.

When used in architectural applications such as the GIS, photogrammetry has several practical benefits over the more traditional measuring and computer modeling approaches. As a result of the digital nature of the images taken, photogrammetry allows for a large amount of dimensional data to be quickly recorded and later accessed in a few images. A digital model can then be directly generated from the photogrammetric data stored in the images. In contrast, in order to generate a computer model from scratch, numerous measurements of a building's details would need to be made to model even the simplest of buildings. The surveyor would need to spend large amounts of time at the site of the building and possibly be required to set up cumbersome scaffolding equipment. Given a photogrammetric model, no measurements need to 
be made beforehand. In fact, the model can be used if measurements are needed for other purposes.

\section{METHODS}

\section{i. Equipment}

In order to conduct a photogrammetric survey of any object, there are three required components: a camera, a targeting system, and photogrammetry software. In short form, a survey can be conducted in three steps. The targets are first placed around an object. Then, a calibrated camera is used to takes photographs of the targets from various angles and positions. Finally, the photographs are used as input data for the photogrammetric software that links the targets appearing in multiple photos to construct a cohesive 3D model.

A modified Nikon D300, shown in Figure 1, was used for this project. All of its internal components are locked into place and functions which would require an inherently unstable lens configuration, such as zoom and autofocus [2], are disabled. The lens and camera were selected to limit the distortion in the images due to the lens and ensure the flatness and regularity of the charged coupled device in the camera [2]. Although amateur cameras are coming into more widespread use [3], for the greatest precision, a metric camera like the modified D300 is preferred. A metric camera gives the advantage of an unchanging geometric configuration of the camera and lens system and thus a known interior orientation [2]. For a non-metric camera, it would be necessary to calibrate the camera before each survey to determine the various distortion parameters (radial, decentering and linear) in the camera and lens. Alternatively, the known parameters of a metric camera can simply be saved and reused between projects.

The bulk of the targets that were used in the photogrammetric survey consist of circular, red (set against a black background), retroreflective targets placed on or around the desired 
object. The variety of targets used is shown in Figure 2. These targets allow the photogrammetric software to quickly pinpoint the location of each target in the photographs within 0.2 inches on average. Within the network of the targets, the position of an object's features can be then established. Previous data shows that the Nikon D300 can obtain greater precision, to within one hundred microns [4], but in the context of a building which has dimensions of several tens of feet, within an inch of precision is acceptable. The shape, color, and retroreflective properties of the targets were specifically selected in order to easily pinpoint the centroid of the targets. It is expected that this pinpointing is possible at camera positions that can view the target at an angle of up to 60 degrees off the central axis of the camera. That is, in Figure 3, the target point $\mathrm{M}$ is located at an angle $i$ of less than 60 degrees from the $\mathrm{Z}$ axis corresponding to the camera axis. Beyond this point, even though the targets may still be seen in the photographs, their reflective property diminishes, resulting in a loss of accuracy.

Australis is a photogrammetry program that was designed to allow for automatic measurements of a targeted object. The software scans each image for retroreflective targets. Some of the targets used have a series of red, reflective dots arranged in a particular pattern, or code, which allows for Australis to recognize identically coded targets in multiple pictures and automatically start creating a 3D target network as shown in Figure 4. Comparatively, without the coded targets, the single red dots would be indistinguishable from each other and would have to be manually referenced between images. With the coded targets, however, the position of the single targets can also be accurately and automatically located in relation to the position of the coded targets, creating a denser target network than would be allowed by the number of coded targets alone. In addition to this network of reflective targets, Australis also allows for the manual marking of feature points and lines (e.g., corners, doors, windows) on the building itself. 
Similar to the determination of the single reflective targets' locations, the building features' positions are accurately found within the realm of the dense 3D target network.

\section{ii. Survey Process}

Once the appropriate materials had been obtained, the photogrammetric survey could be carried out. As an example to study the practicality of photogrammetric modeling, Building 281 on SLAC campus was selected for this survey. The survey was taken in three major phases: camera calibration, survey planning and setup, and modeling in Australis.

Although the camera calibration step can be excluded in later surveys due to the metric nature of the Nikon D300 used, it was necessary to conduct an initial calibration for this preliminary study. The calibration was carried out by placing a large and dense network of retroreflective targets on a flat wall. Images were then taken of the target-covered wall using the camera from various heights, positions and orientations. This process was conducted in the dark so that the only significant light source was from the flash of the camera. By running a set of about 25 images of the calibration wall through Australis, the calibration parameters of the camera were determined. The results of repeat tests were in agreement, giving confirmation of the metric nature of the camera. The parameters that were used for the duration of this study are listed in Table 1.

The next phase was the actually planning and conducting of the photogrammetric survey. After various attempts, a reliable process using the available equipment was determined. A minimalistic approach was taken in an attempt to design a survey would be worth the time and effort compared to the traditional approaches to measuring buildings. To begin, the targets were

placed. Initially, a scattering of single red, reflective targets was placed on the building to create a network. A higher density of targets was devoted to the corners of the building in order to 
reinforce the connection of the two facades forming the corner. The coded targets were then placed. As with the single targets, less emphasis was placed on the long flat sections of the walls compared to more complex areas. To further strengthen the recognition of the corners in the later Australis modeling phase, several targets were placed in the foreground surrounding the building corners. These targets were able to rotate so that the centroids of the targets remained at the exact same coordinates. Without these targets placed around the building, it would have been difficult to angle the camera so that adequate light was able to reflect off of targets on both walls at the corners and thus connect the target networks on each wall. The final set of targets placed were the scale bars that were manufactured such that the centroids of the targets on either end were exactly 30 inches apart (measurable to within 0.02 inches [5]). These scale bars gave the digital 3D model the correct scale in its dimensions. A vertical and a horizontal scale bar were placed on each major wall of the building. An example of a complete target network setup is shown in Figure 5.

One common issue with architectural photogrammetry arises from the presence of obstructions such as trees or other buildings that may block the visibility of the targets and features of the desired building. In the case of Building 281 in this study, the primary concern was the close proximity of another building that limited the distance at which images could be taken and therefore what could be seen by the camera in each image. Various constraints such as this had to be taken into account to determine proper camera positioning for the entire survey before any images could be taken. No set of equations exists to solve every condition that may be faced in any given architectural survey; however, guidelines can be established for the majority of the situations that may be faced. The "3x3" rules for architectural photogrammetry given by [6] can adequately serve such a purpose with some modification and additions made with the 
available equipment in mind. In particular, care must be taken to ensure the appearance of key building features in at least three or more convergent images, and photographs must be taken in a ring of positions surrounding the building. These photographs must also be taken from varying heights, with enough overlap so that all targets on and off the building appear in two or more shots. Although more images would likely provide more redundancy and therefore more precision, it would also require more analysis in Australis.

Once the photographs are taken appropriately, the creation of the $3 \mathrm{D}$ model in Australis is relatively straightforward. The autoreferencing function in the program can create the basis for the 3D model by recognition of the coded targets. A wireframe of the building can then be generated within the context of the reflective target network by manually marking the building's feature points in multiple images.

\section{iii. Feasibility Determination}

There were two equally important primary objectives to this project. The first aim was simply to create a three dimensional wireframe model to show that it was possible with the available equipment and software. Once such a model was generated, the next goal was to determine if it is reasonably accurate so that the photogrammetric survey could serve as a replacement for traditional hand surveying and modeling methods. If a 3D photogrammetric model were to be generated but provided inaccurate data, then it would be of little use.

Achievement of the first goal is easily checkable by merely conducting the photogrammetric survey and attempting to model with it in Australis. Most of the equipment and the Australis software can be tested with several simple surveys. The visibility of the targets was checked by placing the targets as would be done in a normal full building survey. Then, several photographs can be taken from various angles to establish the angles from which the targets are 
identifiable by Australis. A separate set of surveys is, however, necessary for the rotating targets. Rotating targets were used because they can be seen at any angle by being turned to face the camera. However, a test was conducted to ensure that while rotating, the targets maintained the same centroid position. First, a survey was taken of a façade with the normal distribution of coded and point targets placed on it while the rotating targets were spread out in front facing parallel to the wall. Then, two similar surveys were conducted with half of the targets rotated by 45 degrees to either the left or right. After running the images through Australis and setting the same coordinate system based off non-moving point targets on the wall, the positions of all of the points in the survey, including the rotating targets, were compared between the three similar surveys.

The final test of the equipment and Australis was to plan out and conduct a full survey of a building to ascertain whether or not the use of photogrammetry for the generation of a 3D model was a viable and practical approach. Not only was it necessary to find out if the creation of the model was achievable, but also if the model outputted would be accurate enough to serve as an alternative modeling technique for placement in the GIS.

Although Australis does give results on the precision of the target locations by outputting standard errors and root mean squares, to determine the accuracy of the model, it is necessary to still perform a hand survey with a tape measure to obtain the real measurements. Measurements from both the photogrammetric model and the hand survey must be within one inch of agreement to sufficiently demonstrate that the photogrammetric model is valid and accurate. 


\section{RESULTS}

\section{i. Equipment and Software Testing}

Most of the targets were shown to be easily recognizable in Australis at angles up to 60 degrees off the camera axis. Using nine total rotating targets, the standard deviation of the XYZ coordinates of the 5 rotated targets was comparable to the other 193 targets used in the survey as shown in Table 2.

The construction of a complete three dimensional model from a photogrammetric survey of Building 281 was not shown to be easily achievable. After the photographs were taken, Australis was only able to automatically recognize the target network on the first wall. Even with the rotating targets, Australis was simply unable to automatically recognize the sharp corners. From the meager automatically generated model, it required an additional hour to manually reference points between photographs to create a coherent 3D model of the target network shown in Figure 6. However, bearing in mind alternative methods of computer modeling and the accuracy given by this photogrammetric method, this can still be considered to be a fairly rapid process. Once this initial model was created, marking the feature points of the building to generate the actual building model was attempted. About two of the walls were successfully modeled and are shown in Figure 7. However, as the process continued to the third wall, errors began appearing in points that Australis had already previously established in the model. Quickly, the errors caused Australis to deconstruct the model it had originally created as shown in Figure 8. The minor imprecisions associated with the manual marking of hundreds of points in the 3D model seemed to stretch Australis beyond what it was capable of calculating in a reasonable number of iterations. 


\section{ii. Photogrammetry as a Replacement for Traditional Methods}

Despite its inability to create a three dimensional model of the entire building, Australis still seemed able to generate models of the walls separately without excessive difficulty. These partial models can still be used in a GIS if each wall were individually placed at the correct coordinates. Therefore, it was still worthwhile to assess the accuracy of the dimensions from the partial photogrammetric models.

Using a photogrammetrically-generated wireframe model of two exterior walls faces, shown in Figure 7, an error assessment was conducted to compare the dimensions of the photogrammetric model against the true dimensions of the building.

The results shown in Table 3 will show that the photogrammetric model's measurements are within the desired inch of accuracy. However, as a result of the imprecise marking of the building's feature points, the average standard error, as calculated by Australis, is on the range of several feet. As shown in Table 4, some points display a standard error of up to 35 feet, which is greater than some of the dimensions of the building. At best, the standard error of the coordinates is 2.388 inches. Comparably, when given just the reflective target network, the average standard error was 0.144 inches.

\section{DISCUSSION}

With the current equipment and software available, it would not be recommended that photogrammetric surveys be conducted for the purpose of creating entire three dimensional models of buildings. The primary reason for this conclusion is the error prone nature of Australis when constructing the wireframe model. The current available method for photogrammetry requires a large amount of set up time before the survey is conducted. If only several quick measurements are necessary, then measurements by hand could often be taken with more ease. In 
considering the software aspect, Australis is, at the moment, still far too error prone to be used for creating complete models.

However, because the partial 2-wall models were shown to be accurate, the partial models could instead be inserted into the GIS rather than the full model. Since each model is accurate to within an inch, if placed in the correct coordinates, the separate partial models should line up to create a full model. There is still some concern with the calculated standard errors of many of the coordinates; however, this was not shown to have any bearing on the accuracy of the model. Most likely, these standard errors simply prevent the creation of the full model at this point.

Future work on this project would include further attempts to tweak the positioning of targets and camera positions in a way that would allow Australis to automatically recognize the corner. This project showed that Australis is capable of modeling corners. It is hoped that some survey setup will allow the program to accomplish this automatically.

The eventual goal still remains the same, to model the entirety of a building's exteriors in three dimensions. In due course, the interiors can also be modeled similarly. However, it is likely that the capabilities of the Australis software will need further work before this even the modeling of the exteriors can be effectively and reliably achieved.

\section{ACKNOWLEDGEMENTS}

I would like to thank the Department of Energy and the SLAC National Accelerator Laboratory for funding and hosting the SULI program and this project. Special thanks is given to my mentors, Catherine LeCocq and Brian Fuss, for their guidance and knowledge throughout the duration of the project. I would also like to thank all of the SULI students and staff at SLAC for their support. 


\section{REFERENCES}

[1] M.A.R. Cooper and S. Robson, "Theory of close range photogrammetry," in Close Range Photogrammetry and Machine Vision, K.B. Atkinson, Ed., Latheronwheel, Scotland: Whittles, 1996.

[2] J.G. Fryer, "Camera calibration," in Close Range Photogrammetry and Machine Vision, K.B. Atkinson, Ed., Latheronwheel, Scotland: Whittles, 1996.

[3] T. Luhmann, S. Robson, S. Kyle, and I. Harley, Close Range Photogrammetry: Principles, Techniques and Applications, Dunbeath, Scotland: Whittles, 2006.

[4] C.M. LeCocq, Internal Notes, SLAC, 2010.

[5] “iWitnessPRO Scale Bars,” [Online document], [2010 Aug 6], Available at HTTP: http://www.iwitnessphoto.com/products/scale_bar.html

[6] P. Waldhäusl and C. Ogleby, " 3 x 3 rules for simple photogrammetric documentation of architecture," International Archives of Photogrammetry and Remote Sensing, vol. XXX, part 5, pp. 426-429, 1994.

\section{FIGURES}

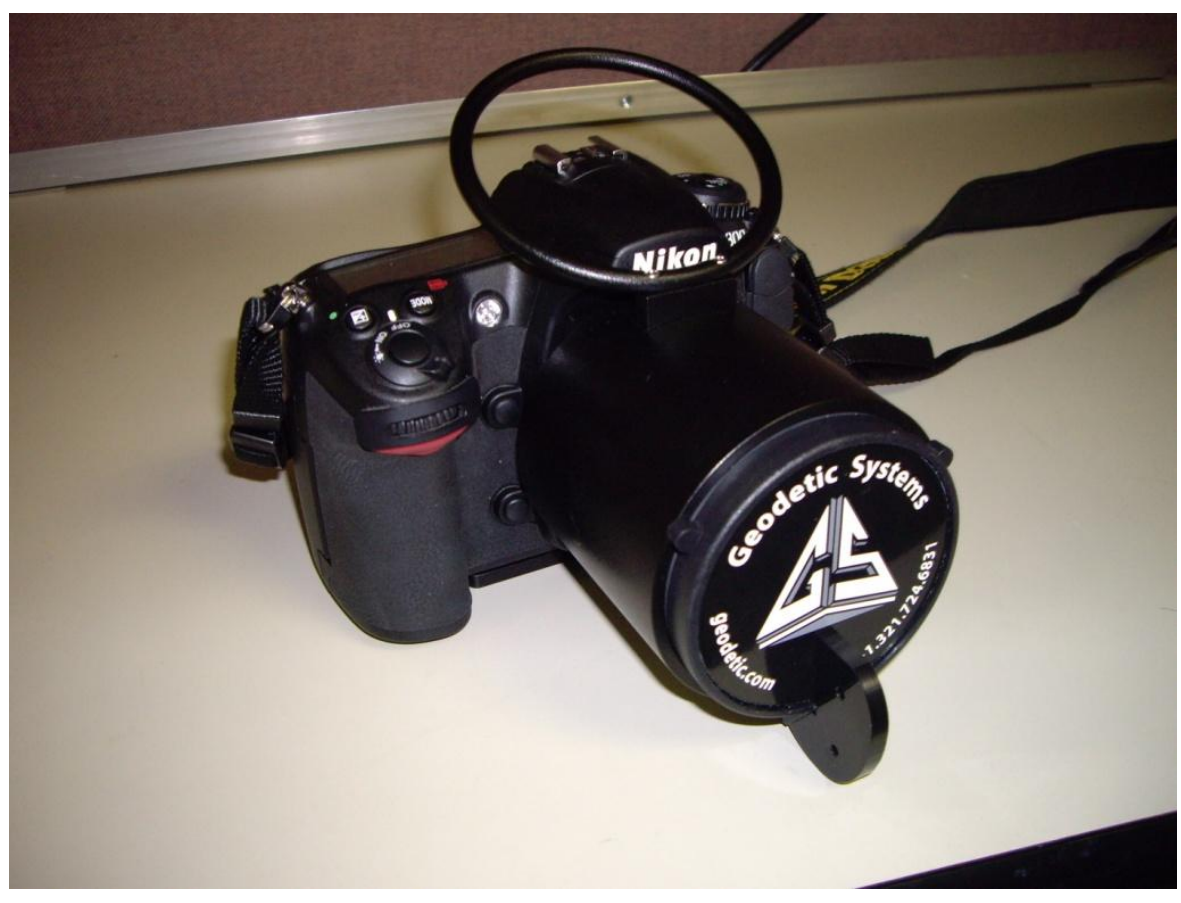

Figure 1 Modified Nikon D300. Several external and internal modifications were made to hold moving parts in place to create a metric camera. 

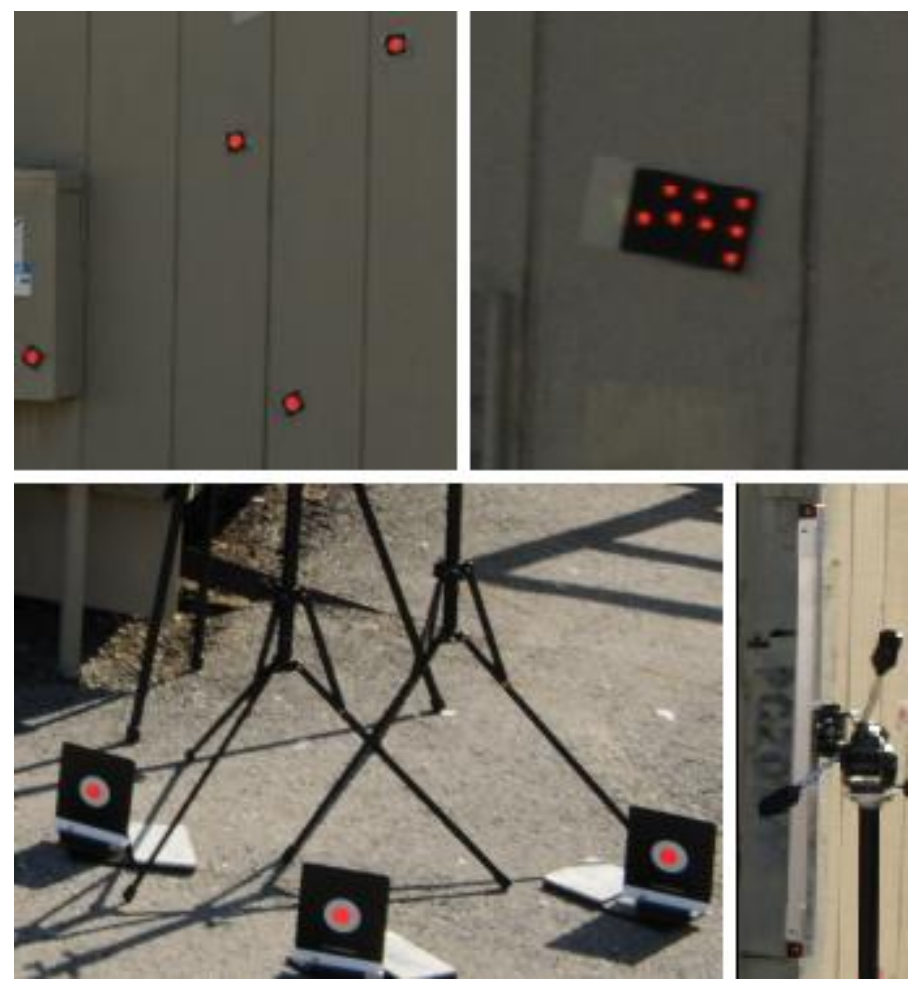

Figure 2 Single circular targets (upper left) are quickly constructed and used to create a dense network. Coded targets (upper right) have a unique pattern recognizable to Australis. Rotating targets (bottom left) can be turned to be viewed at any camera position while maintaining the same centroid position. Scale bars (bottom right) are 30 inches long to give the entire model proper dimensions.

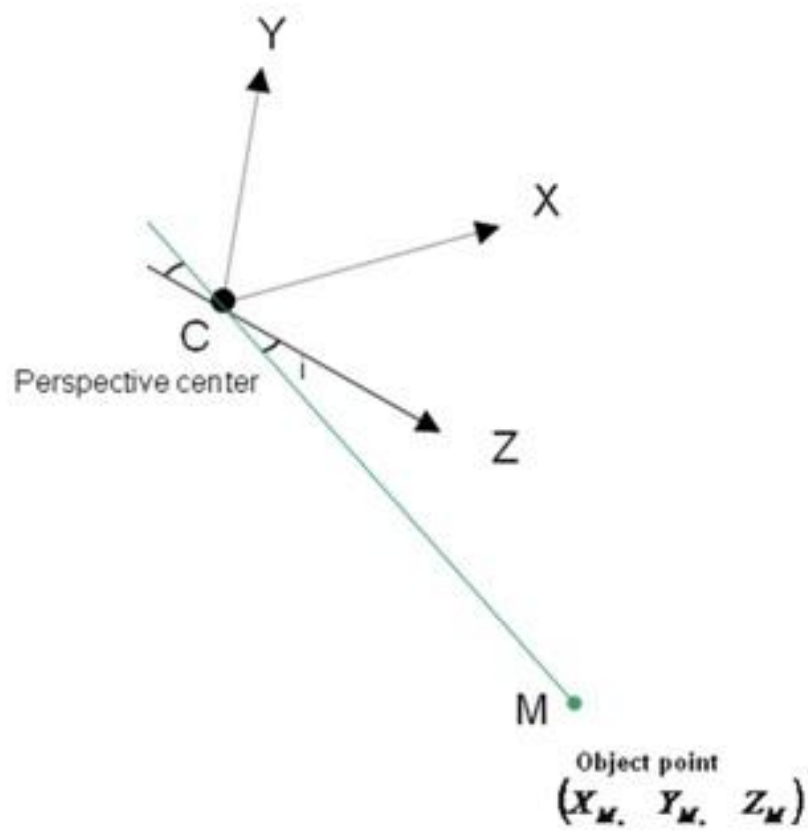

Figure 3 The $Z$ axis is the central axis of the camera. The $X Y$ plane represents the plane of the camer. Object points $M$ at an angle $i$ of less than 60 degrees from the $Z$ axis reflect enough light to view in Australis. 


\section{METRIC CALIBRATION PARAMETERS}

Resolution $=4288 \times 2848$ pixels

Pixel width $=0.0055 \mathrm{~mm}$, Pixel height $=0.0055 \mathrm{~mm}$

\section{VALUE}

\section{STANDARD ERROR}

Principal distance

$\begin{array}{rrr}c= & 18.4801 \mathrm{~mm} & 0.000 \mathrm{~mm} \\ \mathrm{xp}= & -0.0744 \mathrm{~mm} & 0.000 \mathrm{~mm} \\ \mathrm{yp}= & 0.0302 \mathrm{~mm} & 0.000 \mathrm{~mm} \\ \mathrm{~K} 1=5.16825 \mathrm{e}-004 & 2.0104 \mathrm{e}-007 \\ \mathrm{~K} 2=-9.97129 \mathrm{e}-007 & 2.6226 \mathrm{e}-009 \\ \mathrm{~K} 3=-2.19117 \mathrm{e}-009 & 1.0396 \mathrm{e}-011 \\ \mathrm{P} 1 \mid=4.0131 \mathrm{e}-005 & 2.048 \mathrm{e}-007 \\ \mathrm{P} 2=-1.3860 \mathrm{e}-005 & 1.922 \mathrm{e}-007 \\ \mathrm{~B} 1=1.6930 \mathrm{e}-004 & 1.820 \mathrm{e}-006 \\ \mathrm{~B} 2=6.6916 \mathrm{e}-005 & 1.846 \mathrm{e}-006\end{array}$

Table 1 Calibration parameters of the metrically modified Nikon D300. 


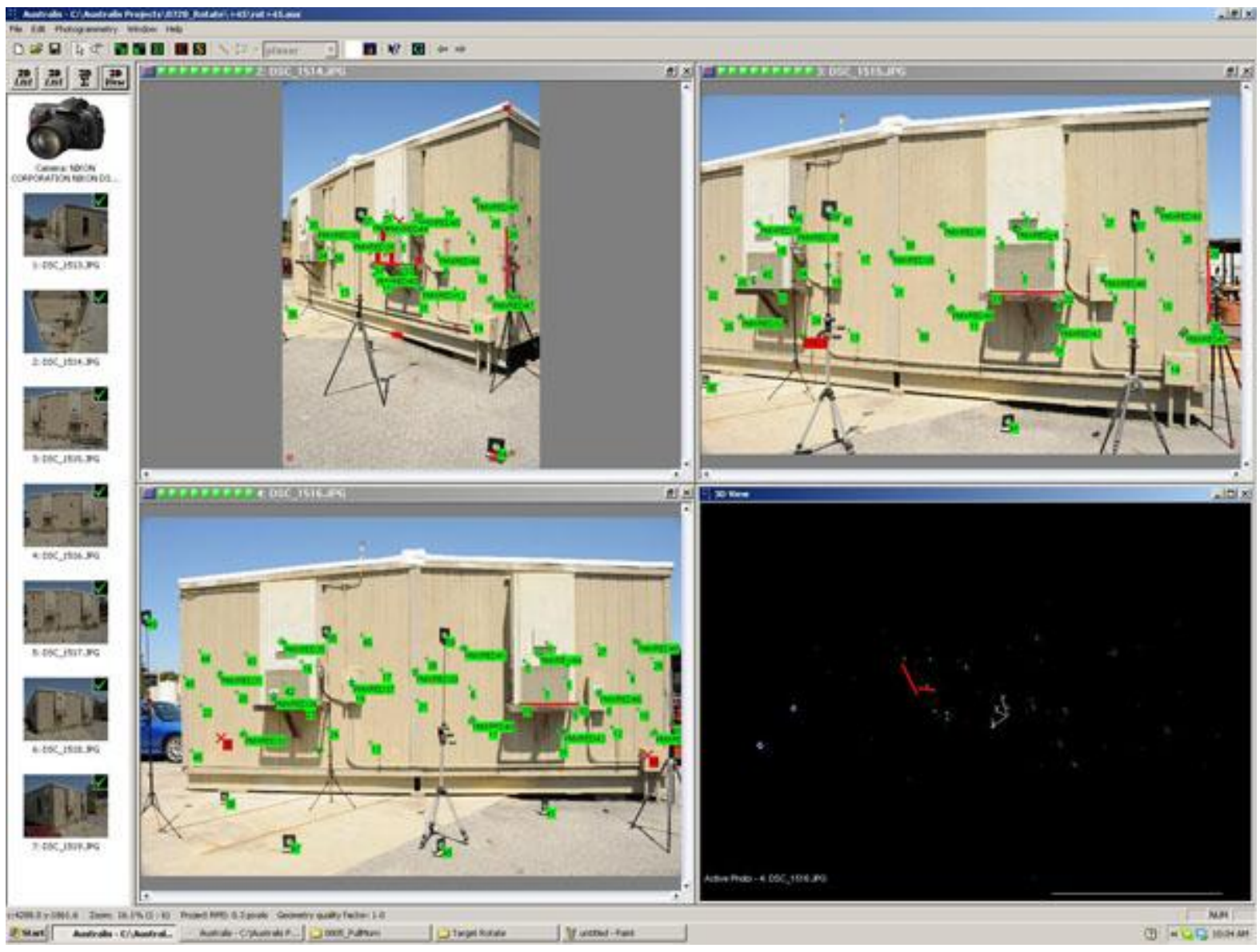

Figure 2 Matching targets are identified from multiple camera positions to create a 3D target network

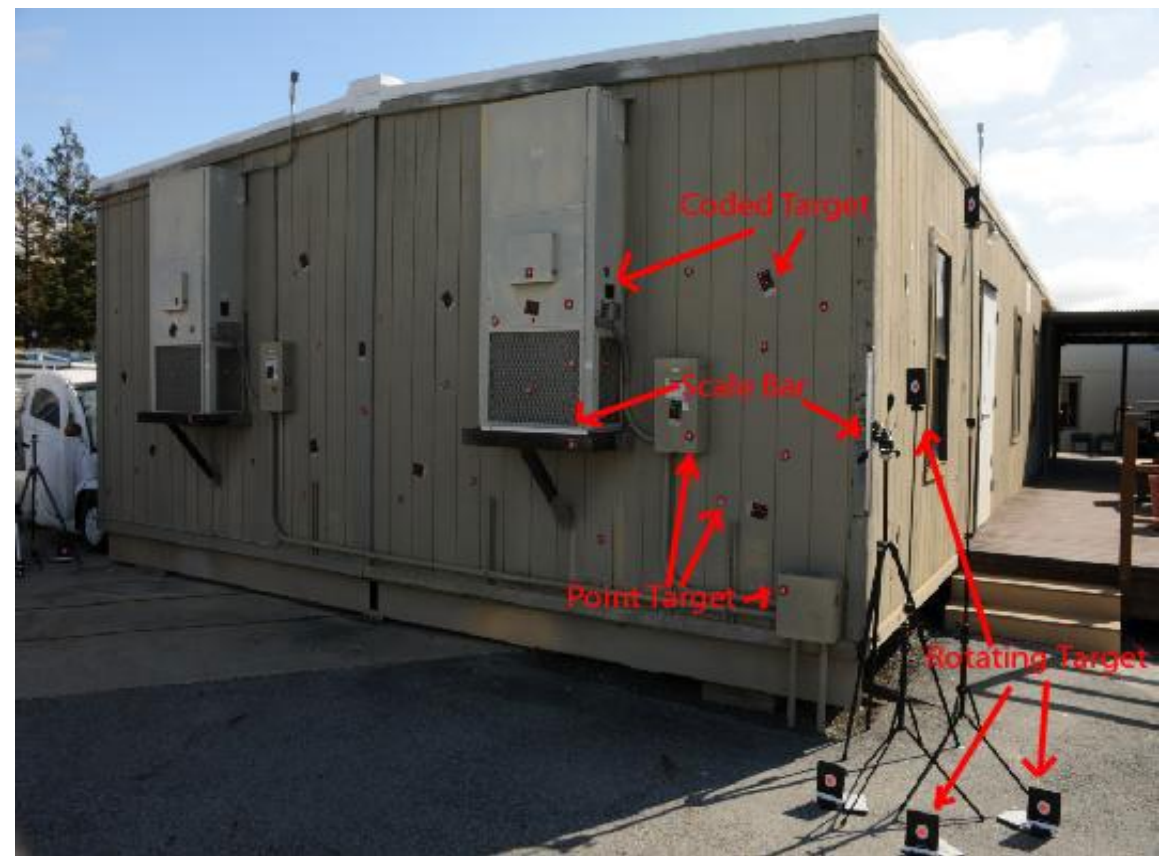

Figure 3 Complete target network on a façade. 


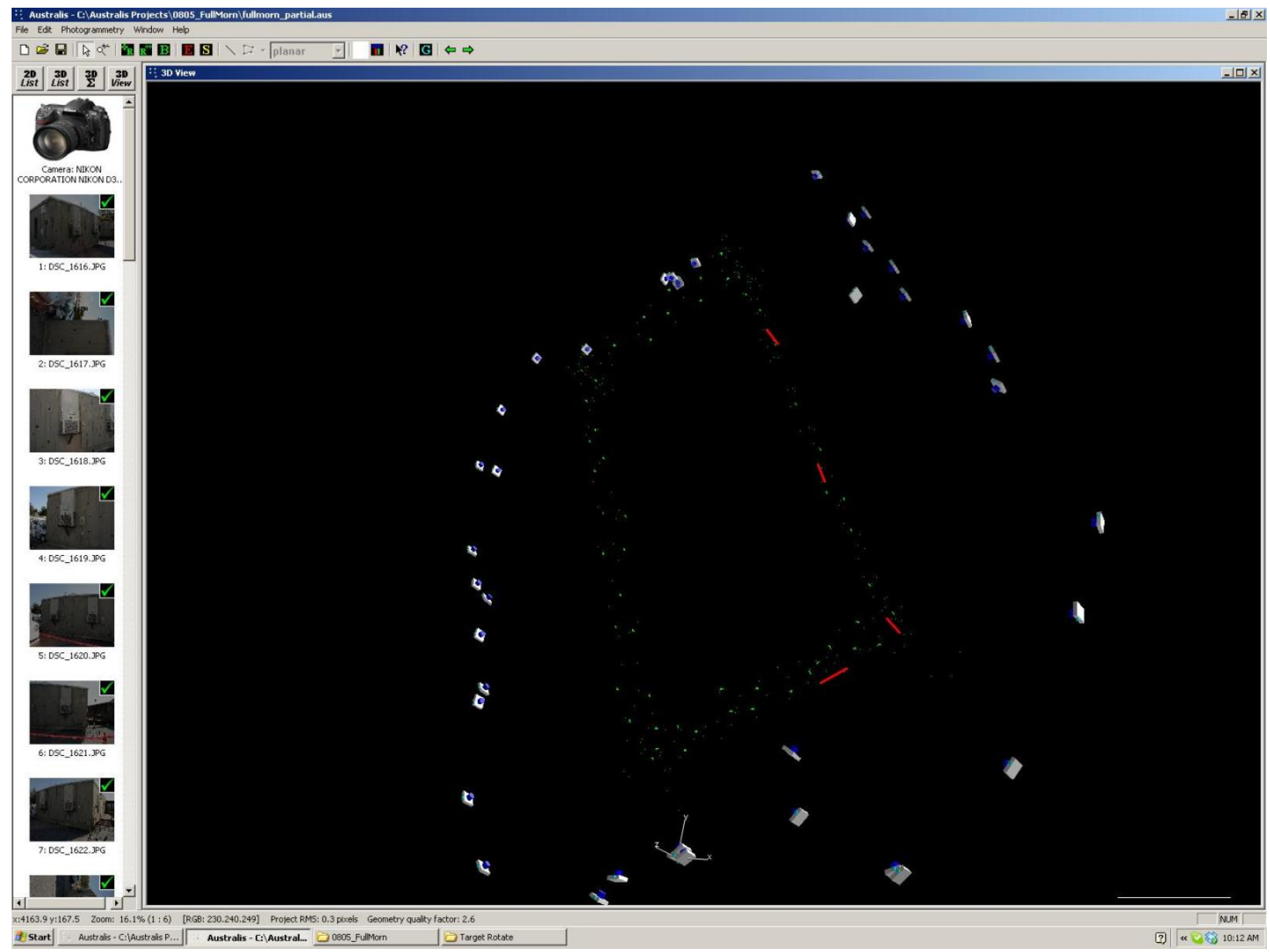

Figure 4 Three dimensional target network on Building 283 photogrammetrically generated in Australis. 


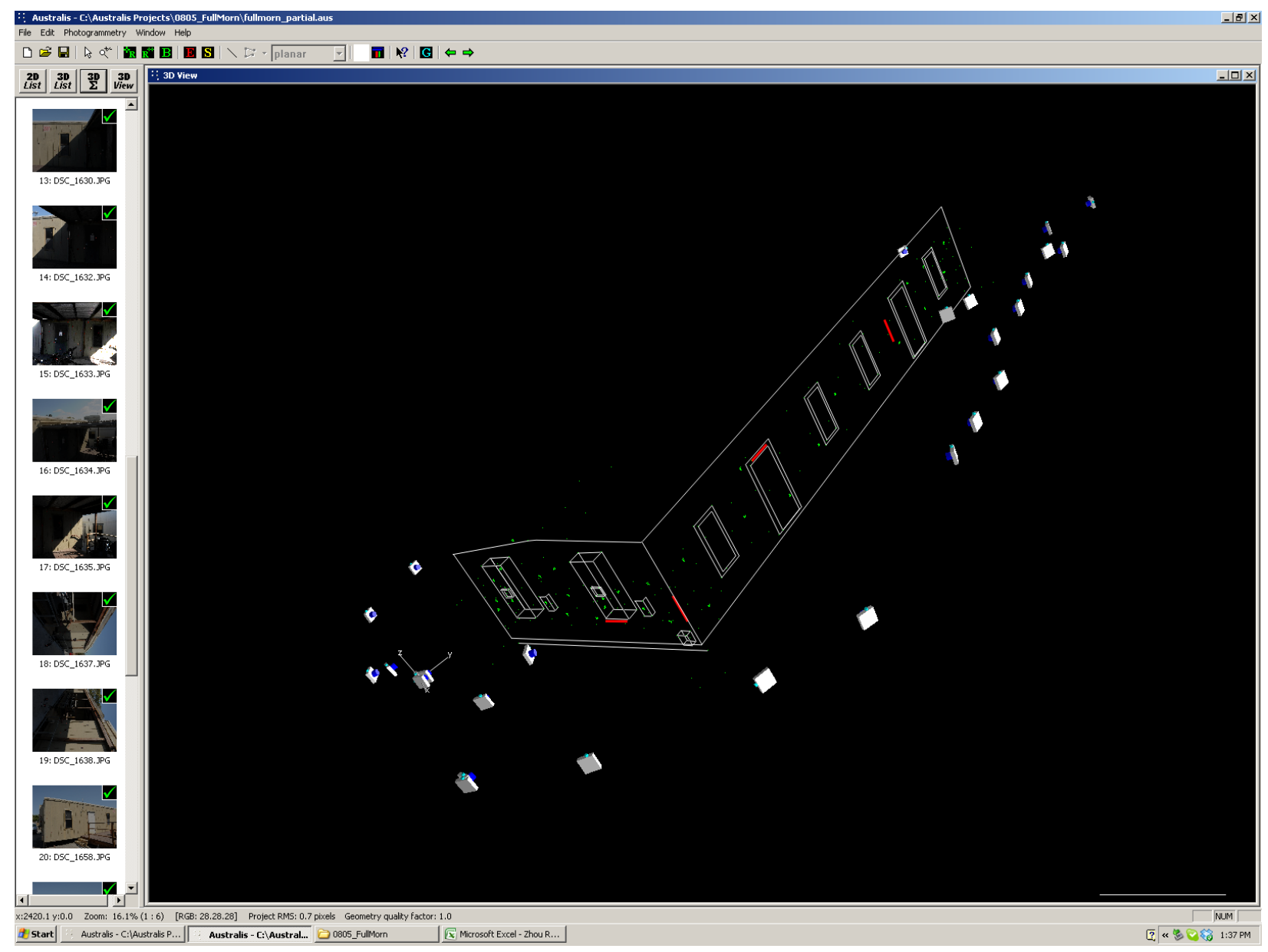

Figure 5 Photogrammetric 3D wireframe model of two exterior wall of SLAC Building 281. 


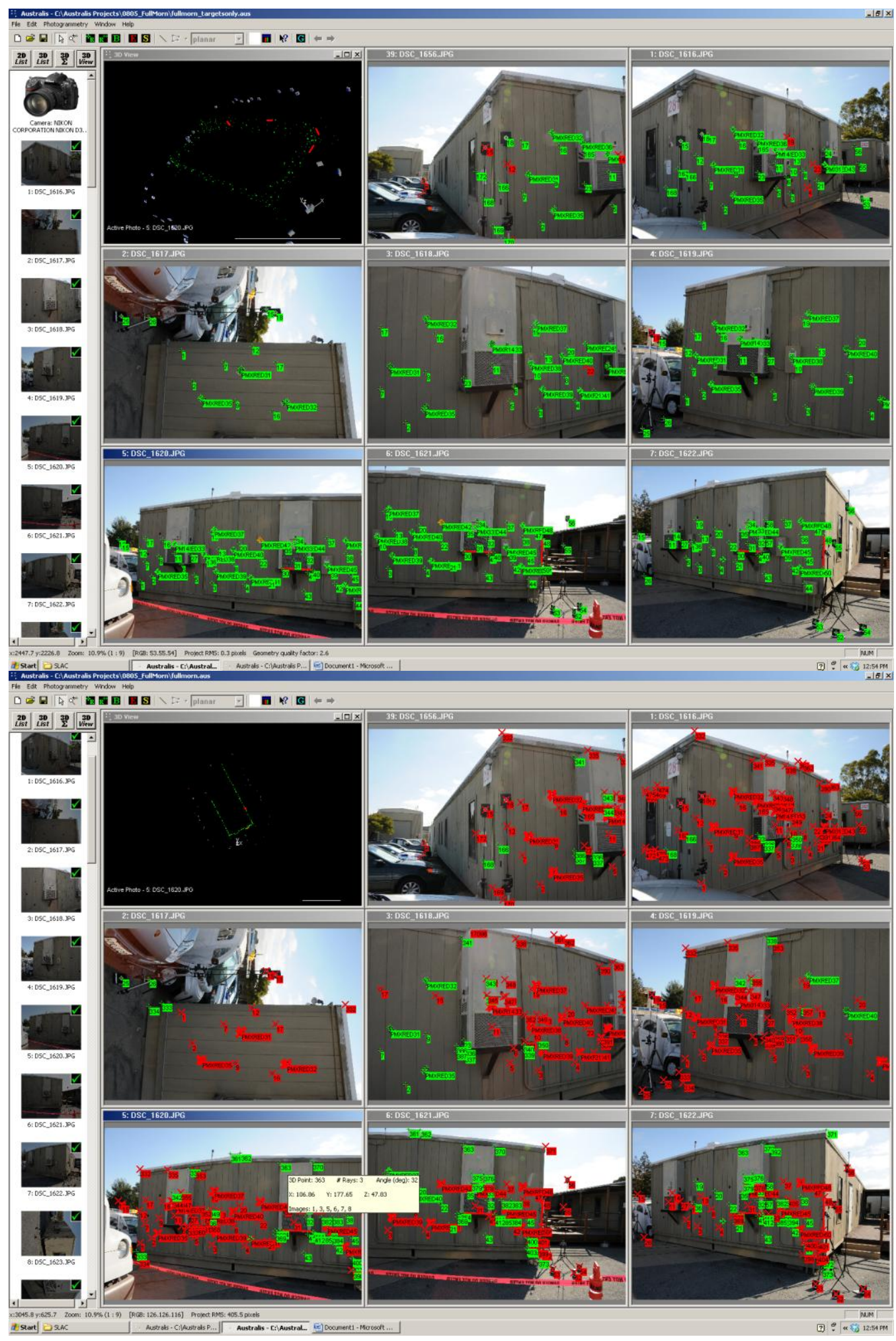

Figure 6 Although Australis has accurately established the a target network for all four exterior walls in the upper screenshot, as more feature points are marked, Australis is no longer able to calculate the position of many of the originally established points. The digital model is deconstructed and the work is irretrievable. 


\begin{tabular}{l|rrr} 
& \multicolumn{3}{|c}{ Standard Deviations (inches) } \\
& Average & Minimum & Maximum \\
\hline Rotating Targets & 0.45 & 0.39 & 0.5 \\
Other Targets & 0.39 & 0 & 1.02
\end{tabular}

Table 2 Standard deviations of the positions of the 5 rotating targets in comparison to the standard deviations of the positions of the 193 stationary targets in a series of photogrammetric surveys of a façade.

\begin{tabular}{l|rrr} 
& \multicolumn{1}{|c}{ Average } & Minimum & Maximum \\
\hline Difference (inches) & 0.22 & 0.0285 & 0.9636 \\
\% error & $0.72 \%$ & $0.01 \%$ & $2.50 \%$
\end{tabular}

Table 3 Differences between 35 actual measurements from hand survey with tape measure and photogrammetric measurements from 3D model.

\begin{tabular}{l|rrr} 
& \multicolumn{3}{|c}{ Standard Errors (inches) } \\
& Average & Minimum & Maximum \\
\hline Targets Only & 0.144 & 0.039 & 0.605 \\
With Features & 19.927 & 2.388 & 426.901
\end{tabular}

Table 4 Standard error, as calculated by Australis, in the position of the points with targets only, as shown in Figure 6, and then with feature points, as shown in Figure 7. 